\title{
RESTRICTION OF SIEGEL MODULAR FORMS TO MODULAR CURVES
}

\author{
CRIS PoOR AND David S. YUen
}

We study homomorphisms from the ring of Siegel modular forms of a given degree to the ring of elliptic modular forms for a congruence subgroup. These homomorphisms essentially arise from the restriction of Siegel modular forms to modular curves. These homomorphisms give rise to linear relations among the Fourier coefficients of a Siegel modular form. We use this technique to prove that $\operatorname{dim} S_{4}^{10}=1$.

\section{INTRONUCTION}

A Siegel modular cusp form of degree $n$ has a Fourier series $f(\Omega)=\sum_{t} a(t) e(\operatorname{tr}(\Omega t))$ where $t$ runs over $\mathcal{X}_{n}$, the set of positive definite semi-integral $n \times n$ forms. If we restrict attention to cusp forms of even weight then the Fourier coefficients are class functions of $t$. The vector space $S_{n}^{k}$ of cusp forms of weight $k$ is finite dimensional and so there exist finite subsets $\mathcal{S} \subset$ classes $\left(\mathcal{X}_{n}\right)$ such that the projection map $\mathrm{FS}_{\mathcal{S}}: S_{n}^{k} \rightarrow \mathbb{C}^{\mathcal{S}}$ given by $f \mapsto \prod_{[t] \in \mathcal{S}} a(t)$ is injective. The following Theorem [13, p. 218] gives one such $\mathcal{S}$ that is readily computable from $n$ and $k$. Instead of ordering semi-integral forms $t$ by their determinant det $(t)$ we order them by their dyadic trace $w(t)$. Denote by $\mathcal{P}_{n}(\mathbb{F})$ the positive definite $n \times n$ symmetric matrices with coefficients in $\mathbb{F} \subseteq \mathbb{R}$. The dyadic trace $w: \mathcal{P}_{n}(\mathbb{R}) \rightarrow \mathbb{R}^{+}$is a class function and only a finite number of classes from $\mathcal{X}_{n}$ will have a dyadic trace below any fixed bound, see [13].

THEOREM 1.1. Let $n, k \in \mathbb{Z}^{+}$. Let

$$
\mathcal{S}=\left\{[t]: t \in \mathcal{X}_{n} \text { and } w(t) \leqslant n \frac{2}{\sqrt{3}} \frac{k}{4 \pi}\right\}
$$

The map FS $\mathcal{S}: S_{n}^{k} \rightarrow \mathbb{C}^{\mathcal{S}}$ is injective.

This Theorem allows one to deduce equality in $S_{n}^{k}$ from equality on the Fourier coefficients for $\mathcal{S}$. There are two obvious avenues for improvement. First, as is evident from Table 1, the bound $\operatorname{dim} S_{n}^{k} \leqslant \operatorname{card}(\mathcal{S})$ is tractable but crude and we would like to trim down the set $\mathcal{S}$ to make card $(\mathcal{S})$ closer to $\operatorname{dim} S_{n}^{k}$. Second, the image $\mathrm{FS}_{\mathcal{S}}(f)$ determines $f$ and one would like to compute some Fourier coefficients outside of $\mathcal{S}$

Received 13th August, 2001

Copyright Clearance Centre, Inc. Serial-fee code: 0004-9727/02 \$A2.00+0.00. 
directly from the Fourier coefficients in $\mathcal{S}$. This paper realises both improvements. We give a method for producing linear relations on the Fourier coefficients of the elements in $S_{n}^{k}$. Table 1 gives $\operatorname{dim} S_{4}^{k}, \operatorname{card}(\mathcal{S})$ and examples of linear relations for even $k \leqslant 12$. These are the only even weights for which $\operatorname{dim} S_{4}^{k}$ is known and the result $\operatorname{dim} S_{4}^{10}=1$ is a new one.

Table 1.

\begin{tabular}{|c|c|c|c|}
\hline$k$ & $\operatorname{dim} S_{4}^{k}$ & $\operatorname{card}(\mathcal{S})$ & linear relations \\
\hline 2 & 0 & 0 & \\
4 & 0 & 0 & \\
6 & 0 & 1 & $a\left(\frac{1}{2} D_{4}\right)=0$ \\
8 & 1 & 2 & $a\left(\frac{1}{2} D_{4}\right)+a\left(\frac{1}{2} A_{4}\right)=0$ \\
10 & 1 & 10 & see equations (3.3) \\
12 & 2 & 23 & 21 uncomputed relations \\
\hline
\end{tabular}

For $k \leqslant 4$ we have $\mathcal{S}=\emptyset$ and so Theorem 1.1 by itself proves $S_{4}^{k}=0$, results due to Christian [2] and Eichler [4, 5]. For $k=6$ we have $\mathcal{S}=\left\{\left[D_{4} / 2\right]\right\}$ and the method in this paper provides the linear relation $a\left(D_{4} / 2\right)=0$ so that we conclude $\operatorname{dim} S_{4}^{6}=0$. For $k=8$ we have $S=\left\{\left[D_{4} / 2\right],\left[A_{4} / 2\right]\right\}$ and the method provides the linear relation $a\left(D_{4} / 2\right)+a\left(A_{4} / 2\right)=0$ showing that $\operatorname{dim} S_{4}^{8} \leqslant 1$. The Schottky form $J$ is in $S_{4}^{8}[9]$ so we have $\operatorname{dim} S_{4}^{8}=1$, see $[14,11,3]$ for these results. For $k=10$ the $\mathcal{S}$ consists of the ten classes in Table 3 and the method provides the nine linearly independent relations given in equation 3.3. We know the cusp form $G_{10}$ is in $S_{4}^{10}$, see [13, p. 232], so that we have $\operatorname{dim} S_{4}^{10}=1$, a result that has been beyond the reach of other methods $[12,3]$. By the work of Erokhin $\operatorname{dim} S_{4}^{12}=2$ is already known, see $[6,7,11]$. Linear relations among Fourier coefficients for semi-integral forms not solely in $\mathcal{S}$ allow the computation of Fourier coefficients outside of $\mathcal{S}$.

The method of producing linear relations on Fourier coefficients from $S_{n}^{k}$ relies on the homomorphisms $\phi_{s}^{*}: S_{n}^{k} \rightarrow S_{1}^{n k}\left(\Gamma_{0}(\ell)\right)$ which exist for any $s \in \mathcal{P}_{n}(\mathbb{Z})$ and any $\ell \in \mathbb{Z}^{+}$with $\ell s^{-1}$ integral. We write elements of $\Gamma_{1}=\operatorname{Sp}_{1}(\mathbb{Z})$ as $\left(\begin{array}{ll}a & b \\ c & d\end{array}\right)$ and define the subgroup $\Gamma_{0}(\ell)$ by $\ell \mid c$ and the subgroup $\Delta_{1}$ by $c=0$. We define $\phi_{s}(\tau)=s \tau$ so that for $f \in M_{n}^{k}$ we have $\left(\phi_{s}^{*} f\right)(\tau)=f(s \tau)$. There are three important points about these homomorphisms:

(1) The image ring $M_{1}\left(\Gamma_{0}(\ell)\right)$ is amenable to computation.

(2) The Fourier coefficients of $\phi_{s}^{*} f$ at each cusp are linear combinations of the Fourier coefficients of $f$, see Proposition 2.3.

(3) There are lots of $n \times n$ integral forms $s$. 
The first point allows us to work out the linear relations among the Fourier coefficients at all cusps of elements in $S_{1}^{n k}\left(\Gamma_{0}(\ell)\right)$. The second point induces linear relations on the Fourier coefficients of elements in $S_{n}^{k}$ from the linear relations on $S_{1}^{n k}\left(\Gamma_{0}(\ell)\right)$. The third point allows us to continue producing linear relations if more are desired.

We illustrate the technique in weights 6 and 8 when the number of Fourier coefficients remains small. Let $f \in S_{4}^{k}$ have the Fourier expansion $f(\Omega)=\sum_{t} a(t) e(\langle\Omega, t\rangle)$ where $\langle\Omega, t\rangle=\operatorname{tr}(\Omega t)$. Let $D_{4}$ represent the $4 \times 4$ form of this root lattice $\left(D_{4}=2 B_{0}\right.$ from Table 3). We compute the Fourier expansion of $\phi_{D_{4}}^{*} f$ in powers of $q=e(\tau)$. For any $s \in \mathcal{P}_{n}(\mathbb{Q})$ we expand $\phi_{s}^{*} f$ into a Fourier series as

$$
\left(\phi_{s}^{*} f\right)(\tau)=\sum_{j \in \mathrm{Q}^{+}}\left(\sum_{t:\langle s, t\rangle=j} a(t)\right) q^{j} .
$$

For simplicity we shall henceforth assume that $k$ is even. If we introduce the notation $\mathcal{V}(j, s, t)=\operatorname{card}\left\{v \in \mathcal{X}_{n}:[v]=[t],\langle v, s\rangle=j\right\}$ then we can write

$$
\left(\phi_{s}^{*} f\right)(\tau)=\sum_{j \in \mathbb{Q}^{+}}\left(\sum_{[t]} \mathcal{V}(j, s, t) a(t)\right) q^{j}
$$

Table 2 is a table of the representation numbers $\mathcal{V}\left(j, D_{4}, t\right)$ for $j \leqslant 7$, omitted entries are zero. See Table 3 for the list of $B_{0}, B_{1}, \ldots, B_{9}$.

Table 2. $\mathcal{V}\left(j, D_{4}, t\right)$.

\begin{tabular}{|c|c|c|c|c|c|c|c|c|c|c|}
\hline $\mathrm{j}$ & $B_{0}$ & $B_{1}$ & $B_{2}$ & $B_{3}$ & $B_{4}$ & $B_{5}$ & $B_{6}$ & $B_{7}$ & $B_{8}$ & $B_{9}$ \\
\hline 4 & 1 & & & & & & & & & \\
5 & 16 & 48 & & & & & & & & \\
6 & 144 & 288 & 216 & 48 & 12 & & & & & \\
7 & 384 & 1488 & 864 & 288 & 144 & 432 & 240 & 288 & 48 & 16 \\
\hline
\end{tabular}

Thus we have the following expansion:

$$
\begin{aligned}
\left(\phi_{D_{4}}^{*} f\right)(\tau)= & a\left(B_{0}\right) q^{4}+\left(16 a\left(B_{0}\right)+48 a\left(B_{1}\right)\right) q^{5} \\
& +\left(144 a\left(B_{0}\right)+288 a\left(B_{1}\right)+216 a\left(B_{2}\right)+48 a\left(B_{3}\right)+12 a\left(B_{4}\right)\right) q^{6} \\
& +\left(384 a\left(B_{0}\right)+1488 a\left(B_{1}\right)+864 a\left(B_{2}\right)+288 a\left(B_{3}\right)+144 a\left(B_{4}\right)+432 a\left(B_{5}\right)\right. \\
& \left.+240 a\left(B_{6}\right)+288 a\left(B_{7}\right)+48 a\left(B_{8}\right)+16 a\left(B_{9}\right)\right) q^{7}+\cdots
\end{aligned}
$$

The function $\phi_{D_{4}}^{*} f \in S_{1}^{4 k}\left(\Gamma_{0}(2)\right)$ is invariant under the Fricke operator because $D_{4}^{-1}$ is equivalent to $D_{4} / 2$, see Proposition 2.2. The ring $M_{1}\left(\Gamma_{0}(2)\right)$ is generated by $E_{2,2}^{-} \in$ $M_{1}^{2}\left(\Gamma_{0}(2)\right)$ and $E_{4,2}^{-} \in M_{1}^{4}\left(\Gamma_{0}(2)\right)$ and the ring of cusp forms is principally generated 
by $C_{8,2}^{+} \in S_{1}^{8}\left(\Gamma_{0}(2)\right)$. The \pm superscript indicates an eigenvalue of \pm 1 under the Fricke operator. In general we define $E_{k, d}^{ \pm}(\tau)=\left(E_{k}(\tau) \pm d^{k / 2} E_{k}(d \tau)\right) /\left(1 \pm d^{k / 2}\right)$ where the $E_{k}(\tau)=1-\left(2 k / B_{k}\right) \sum_{n=1}^{\infty} \sigma_{k-1}(n) q^{n}$ are the Eisenstein series and the $B_{k}$ are given by $t /\left(e^{t}-1\right)=\sum_{k=0}^{\infty} B_{k} t^{k} / k !$. We have $E_{k, d}^{ \pm} \in M_{1}^{k}\left(\Gamma_{0}(d)\right)$ except in the case of $E_{2, d}^{+}$. The Fourier expansions of these generators are given by

$$
\begin{aligned}
& E_{2,2}^{-}(\tau)=1+24 \sum_{n=1}^{\infty}\left(\sigma_{1}(n)-2 \sigma_{1}(n / 2)\right) q^{n}=1+24 q+24 q^{2}+96 q^{3}+24 q^{4}+144 q^{5}+\ldots \\
& E_{4,2}^{-}(\tau)=1-80 \sum_{n=1}^{\infty}\left(\sigma_{3}(n)-4 \sigma_{3}(n / 2)\right) q^{n}=1-80 q-400 q^{2}-2240 q^{3}-2960 q^{4}-\ldots \\
& C_{8,2}^{+}(z)=\frac{1}{256}\left(E_{2,2}^{-}(\tau)^{4}-E_{4,2}^{-}(\tau)^{2}\right)=q-8 q^{2}+12 q^{3}+64 q^{4}-210 q^{5}-96 q^{6}-\ldots
\end{aligned}
$$

The vanishing order of $\phi_{D_{4}}^{*} f$ at the cusp $[I]$ is at least 4 and because $\phi_{D_{4}}^{*} f$ is an eigenfunction of the Fricke operator the vanishing order at the cusp $[J]$ is the same. Thus we have $\left(C_{8,2}^{+}\right)^{4} \mid \phi_{D_{4}}^{*} f$ in $M_{1}\left(\Gamma_{0}(2)\right)$. For $k=6$ this means $\phi_{D_{4}}^{*} f=0$ and so every coefficient in equation 1.3 gives a homogeneous linear relation; in particular we must have $a\left(B_{0}\right)=0$ (or $a\left(D_{4} / 2\right)=0$ ) and hence by Theorem 1.1 we have $S_{4}^{6}=0$. For $k=8$ there is a parameter $c \in \mathbb{C}$ such that

$$
\phi_{D_{4}}^{*} f=c\left(C_{8,2}^{+}\right)^{4}=c\left(q^{4}-32 q^{5}+432 q^{6}-2944 q^{7}+7192 q^{8}+39744 q^{9}-\ldots\right) .
$$

Elimination of the parameter $c$ provides the following 3 linear relations for any $f \in S_{4}^{8}$.

$$
\begin{gathered}
a\left(B_{0}\right)+a\left(B_{1}\right)=0 \\
-24 a\left(B_{0}\right)+24 a\left(B_{1}\right)+18 a\left(B_{2}\right)+4 a\left(B_{3}\right)+a\left(B_{4}\right)=0 ; \\
208 a\left(B_{0}\right)+93 a\left(B_{1}\right)+54 a\left(B_{2}\right)+18 a\left(B_{3}\right)+9 a\left(B_{4}\right)+27 a\left(B_{5}\right) \\
+15 a\left(B_{6}\right)+18 a\left(B_{7}\right)+3 a\left(B_{8}\right)+a\left(B_{9}\right)=0 .
\end{gathered}
$$

As mentioned, the first relation alone, $a\left(D_{4} / 2\right)+a\left(A_{4} / 2\right)=0$ (note $B_{1}=A_{4} / 2$ ), implies that $\operatorname{dim} S_{4}^{8} \leqslant 1$.

For $k=10$ there are parameters $\alpha, \beta \in \mathbb{C}$ such that $\phi_{D_{4}}^{*} f=\left(C_{8,2}^{+}\right)^{4}\left(\alpha\left(E_{2,2}^{-}\right)^{4}+\right.$ $\left.\beta C_{8,2}^{+}\right)$. The element $\left(E_{2,2}^{-}\right)^{2} E_{4,2}^{-}$cannot occur in this representation because it has eigenvalue -1 under the Fricke operator. Elimination of the parameters $\alpha$ and $\beta$ provides two linear relations:

$$
\begin{aligned}
& 224 a\left(B_{0}\right)=184 a\left(B_{1}\right)+18 a\left(B_{2}\right)+4 a\left(B_{3}\right)+a\left(B_{4}\right) \\
& 21376 a\left(B_{1}\right)=-16110 a\left(B_{2}\right)-3916 a\left(B_{3}\right)-1231 a\left(B_{4}\right)-1512 a\left(B_{5}\right)-840 a\left(B_{6}\right) \\
&5) \quad-1008 a\left(B_{7}\right)-168 a\left(B_{8}\right)-56 a\left(B_{9}\right) .
\end{aligned}
$$


In conjunction with Theorem 1.1 these two relations imply $\operatorname{dim} S_{4}^{10} \leqslant 8$ but it will require another homomorphism $\phi_{H}^{*}: S_{4}^{10} \rightarrow S_{1}^{40}\left(\Gamma_{0}(6)\right)$ and a more extensive computation to prove that $\operatorname{dim} S_{4}^{10} \leqslant 1$.

\section{PROPOSITIONS}

We let $\Gamma_{n}=\operatorname{Sp}_{n}(\mathbb{Z})$. We write elements of $\operatorname{Sp}_{n}(\mathbb{R})$ as $\left(\begin{array}{ll}A & B \\ C & D\end{array}\right)$. The group $\mathrm{Sp}_{n}(\mathbb{R})$ acts on functions from the right via $\left(f \mid\left(\begin{array}{ll}A & B \\ C & D\end{array}\right)\right)(\Omega)=\operatorname{det}(C \Omega+D)^{-k} f$ $\left((A \Omega+B)(C \Omega+D)^{-1}\right)$.

PROPOSITION 2.1. Let $n, \ell \in \mathbb{Z}^{+}$. Let $s, \ell s^{-1} \in \mathcal{P}_{n}(\mathbb{Z})$. The map $\phi_{s}^{*}$ : $M_{n}^{k} \rightarrow M_{1}^{n k}\left(\Gamma_{0}(\ell)\right)$ is a graded ring homomorphism.

PROOF: For $\left(\begin{array}{ll}a & b \\ c & d\end{array}\right) \in \mathrm{Sp}_{1}(\mathbb{R})$ we have

$$
\begin{aligned}
\left(\phi_{s}^{*} f \mid\left(\begin{array}{ll}
a & b \\
c & d
\end{array}\right)\right)(\tau) & =(c \tau+d)^{-n k} f\left(\frac{a \tau+b}{c \tau+d} s\right) \\
& =(c \tau+d)^{-n k} f\left((a \tau s+b s)\left(c s^{-1} \tau s+d I\right)^{-1}\right) \\
& =(c \tau+d)^{-n k} f\left(\left(\begin{array}{cc}
a I & b s \\
c s^{-1} & d I
\end{array}\right) \cdot \tau s\right) \\
& =(c \tau+d)^{-n k} \operatorname{det}\left(c s^{-1} \tau s+d I\right)^{k}\left(f \mid\left(\begin{array}{cc}
a I & b s \\
c s^{-1} & d I
\end{array}\right)\right)(\tau s) \\
& =\left(f \underset{k}{f}\left(\begin{array}{cc}
a I & b s \\
c s^{-1} & d I
\end{array}\right)\right)(\tau s) .
\end{aligned}
$$

If we now assume that $\sigma \in \Gamma_{0}(\ell)$ then $c s^{-1}$ is integral and so $\left(\begin{array}{cc}a I & b s \\ c s^{-1} & d I\end{array}\right) \in \operatorname{Sp}_{n}(\mathbb{Z})$. Therefore we have $\left(f \mid\left(\begin{array}{cc}a I & b s \\ c s^{-1} & d I\end{array}\right)\right)(\tau s)=f(\tau s)=\phi_{s}^{*} f(\tau)$. It is straightforward to see that $\phi_{s}^{*} f$ is holomorphic on $\mathcal{H}_{1}$ and that it is bounded on domains of type $\left\{\tau \in \mathcal{H}_{1}: \operatorname{Im} \tau>y_{0}\right\}$. Thus we have $\phi_{s}^{*}: M_{n}^{k} \rightarrow M_{1}^{n k}\left(\Gamma_{0}(\ell)\right)$.

For $\ell \in \mathbb{Z}^{+}$let $W_{\ell}=\left(\begin{array}{cc}0 & -1 \\ \ell & 0\end{array}\right) / \sqrt{\ell}$ denote the Fricke involution.

Proposition 2.2. Let $n, \ell \in \mathbb{Z}^{+}$. Let $s, \ell s^{-1} \in \mathcal{P}_{n}(\mathbb{Z})$. Let $f \in M_{n}^{k}$. Assume that $s$ is $\mathrm{GL}_{n}(\mathbb{Z})$-equivalent to $\ell s^{-1}$. Then $\phi_{s}^{*} f \in M_{1}^{n k}\left(\Gamma_{0}(\ell)\right)$ is an eigenfunction of the Fricke operator $W_{\ell}$. The eigenvalue is +1 unless $s$ is improperly equivalent to $\ell s^{-1}$ and $k$ is odd in which case $\phi_{s}^{*} f$ has eigenvalue -1 under $W_{\ell}$.

Proof: When $s$ is equivalent to $\ell s^{-1}$ we have $U s U^{\prime}=\ell s^{-1}$ for some $U \in$ $\mathrm{GL}_{n}(\mathbb{Z})$. We shall show that $\left(\phi_{s}^{*} f\right) \mid W_{\ell}=\operatorname{det}(U)^{k} \phi_{s}^{*} f$. The factor $\operatorname{det}(U)^{k}$ is one 
except in the case noted. We first check that $\phi_{s} \circ W_{\ell}=\left(\begin{array}{cc}0 & U^{*} \\ -U & 0\end{array}\right) \circ \phi_{s}$. For every $\tau \in \mathcal{H}_{1}$ we have

$$
\begin{aligned}
\left(\phi_{s} \circ W_{\ell}\right)(\tau) & =\phi_{s}\left(-\frac{1}{\ell \tau}\right)=-\frac{1}{\ell} s \tau^{-1}=-U^{*} s^{-1} U^{-1} \tau^{-1}=U^{*}(-U s \tau)^{-1} \\
& =\left(\begin{array}{cc}
0 & U^{*} \\
-U & 0
\end{array}\right)(s \tau)=\left(\left(\begin{array}{cc}
0 & U^{*} \\
-U & 0
\end{array}\right) \circ \phi_{s}\right)(\tau) .
\end{aligned}
$$

Noting that $\left(\begin{array}{cc}0 & U^{*} \\ -U & 0\end{array}\right) \in \Gamma_{n}$ we compute

$$
\begin{aligned}
{\left[\left(\phi_{s}^{*} f\right) \mid W_{n k}\right](\tau) } & =(\sqrt{\ell} \tau)^{-n k}\left(\phi_{s}^{*} f\right)\left(W_{\ell}(\tau)\right) \\
& =(\sqrt{\ell} \tau)^{-n k}\left(f \circ \phi_{s} \circ W_{\ell}\right)(\tau) \\
& =(\sqrt{\ell} \tau)^{-n k}\left(f \circ\left(\begin{array}{cc}
0 & U^{*} \\
-U & 0
\end{array}\right) \circ \phi_{s}\right)(\tau) \\
& =(\sqrt{\ell} \tau)^{-n k} \operatorname{det}(-U s \tau)^{k} f\left(\phi_{s}(\tau)\right) \\
& =(-\sqrt{\ell})^{-n k} \operatorname{det}(U)^{k} \operatorname{det}(s)^{k}\left(\phi_{s}^{*} f\right)(\tau) \\
& =\operatorname{det}(U)^{k}\left(\phi_{s}^{*} f\right)(\tau) .
\end{aligned}
$$

In the last line above we have used the fact that $\operatorname{det}(s)^{2}=\ell^{n}$ and that when $n k$ is odd we must have $f$ identically zero.

The next Proposition shows how to develop the Fourier expansion of $\phi_{s}^{*} f$ at any cusp.

PROPOSITION 2.3. Let $n \in \mathbb{Z}^{+}$. Let $s \in \mathcal{P}_{n}(\mathbb{Q})$. Let $f \in S_{n}^{k}$ have the Fourier expansion $f(\Omega)=\sum_{t} a(t) e(\langle\Omega, t\rangle)$. Let $\left(\begin{array}{ll}a & b \\ c & d\end{array}\right) \in \Gamma_{1}$. There exist $\mathcal{A}, \mathcal{B} \in \mathbb{Q}^{n \times n}$ such that $\left(\begin{array}{cc}a I & b s \\ c s^{-1} & d I\end{array}\right) \in \Gamma_{n}\left(\begin{array}{cc}\mathcal{A} & \mathcal{B} \\ 0 & \mathcal{A}^{*}\end{array}\right)$ and for any such $\mathcal{A}, \mathcal{B}$ we have

$$
\begin{aligned}
\left(\left.\phi_{s}^{*} f\right|_{n k}\left(\begin{array}{ll}
a & b \\
c & d
\end{array}\right)\right)(\tau) & =(\operatorname{det} \mathcal{A})^{k} f\left(\tau \mathcal{A} s \mathcal{A}^{\prime}+\mathcal{B} \mathcal{A}^{\prime}\right) \\
& =(\operatorname{det} \mathcal{A})^{k} \sum_{j \in \mathbb{Q}^{+}}\left(\sum_{t:\left\langle\mathcal{A} s \mathcal{A}^{\prime}, t\right\rangle=j} a(t) e\left(\left\langle t, \mathcal{B} \mathcal{A}^{\prime}\right\rangle\right)\right) q^{j}
\end{aligned}
$$

ProOF: We now wish to study $\left(\phi_{s}^{*} f \mid\left(\begin{array}{ll}a & b \\ c & d\end{array}\right)\right)$ for $\left(\begin{array}{ll}a & b \\ c & d\end{array}\right) \in \operatorname{Sp}_{1}(\mathbb{Z})$. Then as in the proof of Proposition 2.1 we have

$$
\left(\phi_{s}^{*} f||_{n k}\left(\begin{array}{ll}
a & b \\
c & d
\end{array}\right)\right)(\tau)=\left(\left.f\right|_{k}\left(\begin{array}{cc}
a I & b s \\
c s^{-1} & d I
\end{array}\right)\right)(\tau s) .
$$


Now, we can always decompose any matrix in $\mathrm{Sp}_{n}(\mathbb{Q})$ as something in $\operatorname{Sp}_{n}(\mathbb{Z})$ times something in $\mathrm{Sp}_{n}(\mathbb{Q})$ with $C=0[8, \mathrm{p} .125]$. So let $\left(\begin{array}{cc}a I & b s \\ c s^{-1} & d I\end{array}\right) \in \Gamma_{n}\left(\begin{array}{cc}\mathcal{A} & \mathcal{B} \\ 0 & \mathcal{A}^{*}\end{array}\right)$. Since $f$ is automorphic with respect to $\Gamma_{n}$ we have

$$
\begin{aligned}
\left(\phi_{s}^{*} f \mid \underset{n k}{\mid}\left(\begin{array}{ll}
a & b \\
c & d
\end{array}\right)\right)(\tau) & =\left(\left.f\right|_{k}\left(\begin{array}{cc}
a I & b s \\
c s^{-1} & d I
\end{array}\right)\right)(\tau s) \\
& =\left(\left.f\right|_{k}\left(\begin{array}{cc}
\mathcal{A} & \mathcal{B} \\
0 & \mathcal{A}^{*}
\end{array}\right)\right)(\tau s) \\
& =(\operatorname{det} \mathcal{A})^{k} f\left(\tau \mathcal{A} s \mathcal{A}^{\prime}+\mathcal{B A}^{\prime}\right) .
\end{aligned}
$$

The Fourier expansion for $\left(\phi_{s}^{*} f \mid\left(\begin{array}{ll}a & b \\ c & d\end{array}\right)\right)(\tau)$ follows from the Fourier expansion for $f$ under the substitution $\Omega=\tau \mathcal{A} s \mathcal{A}^{\prime}+\mathcal{B} \mathcal{A}^{\prime}$.

The above Proposition provides for the computation of the Fourier expansion of $\phi_{s}^{*} f \mid \sigma$ in general. When $\ell$ is squarefree however the computation of the character $e\left(\left\langle t, \mathcal{B A}^{\prime}\right\rangle\right)$ may be finessed. We introduce a new notation: Notice that $\mathcal{A}$ in Proposition 2.3 is determined up to $u \mathcal{A}$ with $u \in \mathrm{GL}_{n}(\mathbb{Z})$. Thus $\mathcal{A} s \mathcal{A}^{\prime}$ is determined up to equivalence class. We define

$$
s \square\left(\begin{array}{ll}
a & b \\
c & d
\end{array}\right)=\mathcal{A} s \mathcal{A}^{\prime}
$$

with the understanding that this is well-defined only up to equivalence class. Since $f$ is automorphic with respect to $\left(\begin{array}{cc}u & 0 \\ 0 & u^{*}\end{array}\right)$, we have $f\left(u s u^{\prime} \tau\right)=f(s \tau)$ and it makes sense to talk about $f\left(\left(s \square\left(\begin{array}{ll}a & b \\ c & d\end{array}\right)\right) \tau\right)$ and $\phi_{s \square\left(\begin{array}{ll}a & b \\ c & d\end{array}\right)} f$.

Proposition 2.4. Let $s \in \mathcal{P}_{n}(\mathbb{Z})$. Let $\ell \in \mathbb{Z}^{+}$such that $\ell s^{-1}$ is integral and primitive. Let $\left(\begin{array}{ll}a & b \\ c & d\end{array}\right) \in \Gamma_{1}$. Suppose $\operatorname{gcd}(c,(\ell / c))=1$. Let $\widehat{c} \in \mathbb{Z}$ such that $\widehat{c} c \equiv 1$ $\bmod \ell / c$. For any $\mathcal{A}$ with $\left(\begin{array}{cc}a I & b s \\ c s^{-1} & d I\end{array}\right) \in \Gamma_{n}\left(\begin{array}{cc}\mathcal{A} & \mathcal{B} \\ 0 & \mathcal{A}^{*}\end{array}\right)$ we have

$$
\left(\phi_{s}^{*} f \mid\left(\begin{array}{ll}
a & b \\
c & d
\end{array}\right)\right)(\tau)=(\operatorname{det} \mathcal{A})^{k} \phi_{s \square\left(\begin{array}{ll}
a & b \\
c d
\end{array}\right)} f(\tau+d \mathcal{c}) .
$$

Proof: We have $\left(\begin{array}{cc}\mathcal{A} & \mathcal{B} \\ 0 & \mathcal{A}^{*}\end{array}\right)\left(\begin{array}{cc}a I & b s \\ c s^{-1} & d I\end{array}\right)^{-1} \in \mathrm{Sp}_{n}(\mathbb{Z})$. Thus we have

$$
\left(\begin{array}{cc}
\mathcal{A} & \mathcal{B} \\
0 & \mathcal{A}^{*}
\end{array}\right)\left(\begin{array}{cc}
d I & -b s \\
-c s^{-1} & a I
\end{array}\right)=\left(\begin{array}{cc}
d \mathcal{A}-c \mathcal{B} s^{-1} & -b \mathcal{A} s+a \mathcal{B} \\
-c \mathcal{A}^{*} s^{-1} & a \mathcal{A}^{*}
\end{array}\right) \in \mathrm{Sp}_{n}(\mathbb{Z})
$$


Note that each of the four blocks must be in $\mathbb{Z}^{n \times n}$. Multiplying $d \mathcal{A}-c \mathcal{B s}^{-1}$ by the integral $s$ implies $d \mathcal{A} s-c \mathcal{B}$ is integral. Both $\mathcal{A} s$ and $\mathcal{B}$ are integral because we have

$$
\begin{aligned}
\mathcal{A} s & =a(d \mathcal{A} s-c \mathcal{B})+c(-b \mathcal{A} s+a \mathcal{B}), \\
\mathcal{B} & =b(d \mathcal{A} s-c \mathcal{B})+d(-b \mathcal{A} s+a \mathcal{B}) .
\end{aligned}
$$

Since $c \mathcal{B s}^{-1}=(c / \ell) \mathcal{B} \ell s^{-1}$ and $\ell s^{-1} \in \mathbb{Z}^{n \times n}$, we have $c \mathcal{B} s^{-1} \in(c / \ell) \mathbb{Z}^{n \times n}$. This combined with $d \mathcal{A}-c \mathcal{B s}^{-1} \in \mathbb{Z}^{n \times n}$ implies $d \mathcal{A} \in(c / \ell) \mathbb{Z}^{n \times n}$. Also we have $\mathcal{A}=$ $(1 / \ell)(\mathcal{A} s) \ell s^{-1} \in(1 / \ell) \mathbb{Z}^{n \times n}$ and consequently $\mathcal{A}=a(d \mathcal{A})-b(c \mathcal{A}) \in(c / \ell) \mathbb{Z}^{n \times n}$. Since $\mathcal{A} s$ is integral, its transpose $s \mathcal{A}^{\prime}$ is also integral. Then multiplying $d \mathcal{A}-c \mathcal{B s}^{-1}$ by the integral $\widehat{c} s A^{\prime}$ implies that $d \widehat{c} \mathcal{A} s \mathcal{A}^{\prime}$ and $\widehat{c} c \mathcal{B} \mathcal{A}^{\prime}$ differ by an integer matrix. But $\widehat{c} c \equiv 1$ $\bmod (\ell / c)$ and $\mathcal{B} \mathcal{A}^{\prime} \in(c / \ell) \mathbb{Z}^{n \times n}$ imply that $\widehat{c} c \mathcal{B} \mathcal{A}^{\prime}$ and $\mathcal{B} \mathcal{A}^{\prime}$ differ by an integer matrix. Hence $d \widehat{c} \mathcal{A} s \mathcal{A}^{\prime}$ and $\mathcal{B} \mathcal{A}^{\prime}$ differ by an integer matrix. Finally, from Proposition 2.3 we have

$$
\begin{aligned}
& (\operatorname{det} \mathcal{A})^{-k}\left(\phi_{s}^{*} f \mid\left(\begin{array}{ll}
a & b \\
c & d
\end{array}\right)\right)(\tau)=f\left(\tau \mathcal{A} s \mathcal{A}^{\prime}+\mathcal{B} \mathcal{A}^{\prime}\right) \\
& =f\left(\tau \mathcal{A} s \mathcal{A}^{\prime}+d \widehat{c} \mathcal{A} s \mathcal{A}^{\prime}\right) \\
& =f\left(\mathcal{A} s \mathcal{A}^{\prime}(\tau+d \tilde{c})\right) \\
& =\phi_{s \square\left(\begin{array}{ll}
a & b \\
c & d
\end{array}\right)}^{*} f(\tau+d \widetilde{c}) \text {. }
\end{aligned}
$$

\section{THE SPACE $S_{4}^{10}$}

We shall apply the technique of the Introduction to $S_{4}^{10}$. Theorem 1.1 says a form in $S_{4}^{10}$ is determined by its coefficients $a(t)$ with $w(t) \leqslant 3.5$. Table 3 gives the list of these 10 quadratic forms, see $[\mathbf{1 0}, \mathbf{1 3}]$. For uniformity of notation we shall refer to these as $B_{0}, \ldots, B_{9}$. Here the number under $\ell$ for $B_{i}$ is the smallest positive integer such that $\ell\left(2 B_{i}\right)^{-1}$ is integral.

We shall apply the technique to $H=2 B_{4}$ for which $6 H^{-1}$ is integral. By Proposition 1.1 we have $\operatorname{Im} \phi_{H}^{*} f \subset M_{1}\left(\Gamma_{0}(6)\right)$ and our calculations will occur inside this ring. The ring $M_{1}\left(\Gamma_{0}(6)\right)$ is generated by three forms $A, B, C$ of weight 2 . There is one relation $C^{2}=9 B^{2}-8 A^{2}$. The ring of cusp forms is principally generated by a form of weight $4, D=\left(A^{2}-B^{2}\right) / 4$. There are 4 cusps in $\Gamma_{0}(6) \backslash \Gamma_{1} / \Delta_{1}$, represented by $I$, $\sigma_{2}=\left(\begin{array}{ll}1 & 0 \\ 2 & 1\end{array}\right), \sigma_{3} \doteq\left(\begin{array}{ll}1 & 0 \\ 3 & 1\end{array}\right)$ and $J$ with respective widths $1,3,2$ and 6 . We now give the Fourier expansions of the generators at all four cusps. The definition of $E_{2,2}^{-}$ has already been given, similarly define

$$
E_{2,3}^{-}(\tau)=1+12 \sum_{n=1}^{\infty}\left(\sigma_{1}(n)-3 \sigma_{1}(n / 3)\right) q^{n}=1+12\left(q+3 q^{2}+q^{3}+7 q^{4}+6 q^{5}+\cdots\right) .
$$


Define the following elements in $M_{1}\left(\Gamma_{0}(6)\right)$ :

$$
\begin{aligned}
& A(\tau)=(3 / 4) E_{2,2}^{-}(3 \tau)+(1 / 4) E_{2,2}^{-}(\tau)=1+6 q+6 q^{2}+42 q^{3}+\cdots \\
& B(\tau)=(2 / 3) E_{2,3}^{-}(2 \tau)+(1 / 3) E_{2,3}^{-}(\tau)=1+4 q+20 q^{2}+4 q^{3}+\cdots \\
& C(\tau)=(3 / 2) E_{2,2}^{-}(3 \tau)-(1 / 2) E_{2,2}^{-}(\tau)=1-12 q-12 q^{2}-12 q^{3}+\cdots
\end{aligned}
$$

\begin{tabular}{|c|c|c|c|c|}
\hline Name & Form & Dyadic trace & 16. Determinant & $\ell$ \\
\hline$B_{0}$ & $\frac{1}{2}\left(\begin{array}{llll}2 & 1 & 1 & 1 \\
1 & 2 & 0 & 0 \\
1 & 0 & 2 & 0 \\
1 & 0 & 0 & 2 \\
\end{array}\right.$ & 2 & 4 & 2 \\
\hline$B_{1}$ & 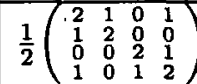 & 2.5 & 5 & 5 \\
\hline$B_{2}$ & $\frac{1}{2}\left(\begin{array}{llll}2 & 0 & 0 & 1 \\
0 & 2 & 0 & 1 \\
0 & 0 & 2 & 0 \\
1 & 1 & 0 & 2\end{array}\right)$ & 3 & 8 & 4 \\
\hline$B_{3}$ & $\frac{1}{2}\left(\begin{array}{llll}2 & 1 & 0 & 0 \\
1 & 2 & 0 & 0 \\
0 & 0 & 2 & 1 \\
0 & 0 & 1 & 2 \\
\end{array}\right)$ & 3 & 9 & 3 \\
\hline$B_{4}$ & $\frac{1}{2}\left(\begin{array}{llll}2 & 1 & 1 & 1 \\
1 & 2 & 0 & 0 \\
1 & 0 & 2 & 0 \\
1 & 0 & 0 & 4\end{array}\right)$ & 3 & 12 & 6 \\
\hline$B_{5}$ & $\frac{1}{2}\left(\begin{array}{llll}2 & 0 & 0 & 1 \\
0 & 2 & 0 & 0 \\
0 & 0 & 2 & 0 \\
1 & 0 & 0 & 2\end{array}\right)$ & 3.5 & 12 & 6 \\
\hline$B_{6}$ & $\frac{1}{2}\left(\begin{array}{llll}2 & 1 & 1 & 0 \\
1 & 2 & 0 & 1 \\
1 & 0 & 2 & 0 \\
0 & 1 & 0 & 4\end{array}\right)$ & 3.5 & 13 & 13 \\
\hline$B_{7}$ & $\frac{1}{2}\left(\begin{array}{llll}2 & 1 & 0 & 1 \\
1 & 2 & 0 & 0 \\
0 & 0 & 2 & 1 \\
1 & 0 & 1 & 4\end{array}\right)$ & 3.5 & 17 & 17 \\
\hline$B_{8}$ & $\frac{1}{2}\left(\begin{array}{llll}2 & 0 & 0 & 1 \\
0 & 2 & 0 & 1 \\
0 & 0 & 2 & 1 \\
1 & 1 & 1 & 4 \\
\end{array}\right.$ & 3.5 & 20 & 10 \\
\hline$B_{9}$ & $\frac{1}{2}\left(\begin{array}{llll}2 & 1 & 1 & 1 \\
1 & 2 & 0 & 1 \\
1 & 0 & 4 & 2 \\
1 & 1 & 2 & 4\end{array}\right)$ & 3.5 & 25 & 5 \\
\hline
\end{tabular}

Table 3. Semi-integral quaternary forms with dyadic trace $\leqslant 3.5$.

The elliptic modular forms $A, B, C$ transform nicely as

$$
\begin{array}{rrr}
(A \mid J)(\tau)=-\frac{1}{6} A(\tau / 6), & \left(A \mid \sigma_{2}\right)(\tau) & =+\frac{1}{3} A((\tau-1) / 3) \\
\left(A \mid \sigma_{3}\right)(\tau) & =-\frac{1}{2} A((\tau-1) / 2) \\
(B \mid J)(\tau)=-\frac{1}{6} B(\tau / 6), & \left(B \mid \sigma_{2}\right)(\tau) & =-\frac{1}{3} B((\tau-1) / 3) \\
\left(B \mid \sigma_{3}\right)(\tau) & =+\frac{1}{2} B((\tau-1) / 2) \\
(C \mid J)(\tau)=+\frac{1}{6} C(\tau / 6), & \left(C \mid \sigma_{2}\right)(\tau) & =-\frac{1}{3} C((\tau-1) / 3) \\
& \left(C \mid \sigma_{3}\right)(\tau) & =-\frac{1}{2} C((\tau-1) / 2)
\end{array}
$$


We use Propostions 2.3 and 2.4 to work out the Fourier expansion of $\phi_{H}^{*} f \mid \sigma$ for $\sigma=I$, $\sigma_{2}, \sigma_{3}, J$. We implement the algorithms from [8, pp. 125, 322-328] to produce a factorisation $\left(\begin{array}{cc}a I & b H \\ c H^{-1} & d I\end{array}\right) \in \Gamma_{n}\left(\begin{array}{cc}\mathcal{A} & \mathcal{B} \\ 0 & \mathcal{A}^{*}\end{array}\right)$ and obtain $\operatorname{det}(\mathcal{A})$ and $H \square \sigma=\mathcal{A} H \mathcal{A}^{\prime}$. We display $H \square \sigma_{2}, H \square \sigma_{3}, H \square J$ and mention that the associated $|\operatorname{det}(\mathcal{A})|$ equals 3 , 4,12 , respectively:

$$
\begin{aligned}
& H \square \sigma_{2}=\frac{1}{3}\left(\begin{array}{cccc}
4 & 2 & 1 & -1 \\
2 & 4 & -1 & 1 \\
1 & -1 & 4 & -1 \\
-1 & 1 & -1 & 4
\end{array}\right) ; \\
& H \square \sigma_{3}=\frac{1}{2}\left(\begin{array}{cccc}
2 & 0 & 0 & 1 \\
0 & 2 & 0 & 1 \\
0 & 0 & 2 & 1 \\
1 & 1 & 1 & 3
\end{array}\right) ; \\
& H \square J=\frac{1}{6}\left(\begin{array}{cccc}
2 & 1 & 1 & 1 \\
1 & 5 & -1 & 2 \\
1 & -1 & 5 & 2 \\
1 & 2 & 2 & 5
\end{array}\right) .
\end{aligned}
$$

Note that all three of the cases $\sigma_{2}, \sigma_{3}, J$ satisfy the hypotheses of Proposition 2.4. Note that for $c=2$, we can take $\widehat{c}=-1$ so that $c \widehat{c}=1 \bmod 3$; for $c=3$, we can take $\widehat{c}=-1$ so that $c \hat{c}=1 \bmod 2$. Thus we have

$$
\begin{aligned}
\left(\phi_{H}^{*} f \mid I\right)(\tau) & =\phi_{H}^{*} f(\tau), \\
\left(\phi_{H}^{*} f \mid \sigma_{2}\right)(\tau) & =3^{-10} \phi_{H \square \sigma_{2}}^{*} f(\tau-1), \\
\left(\phi_{H}^{*} f \mid \sigma_{3}\right)(\tau) & =4^{-10} \phi_{H \square \sigma_{3}}^{*} f(\tau-1), \\
\left(\phi_{H}^{*} f \mid J\right)(\tau) & =12^{-10} \phi_{H \square J}^{*} f(\tau) .
\end{aligned}
$$

Hence the Fourier expansions may be computed from the numbers $\mathcal{V}(j, H \square \sigma, t)$ given in Tables 4, 5, 6 and 7. Among the computations we perform, the computation of these representation numbers is by far the most expensive.

Table 4. $\mathcal{V}(j, H, t)$

\begin{tabular}{|c|c|c|c|c|c|c|c|c|c|c|}
\hline $\mathrm{j}$ & $B_{0}$ & $B_{1}$ & $B_{2}$ & $B_{3}$ & $B_{4}$ & $B_{5}$ & $B_{6}$ & $B_{7}$ & $B_{8}$ & $B_{9}$ \\
\hline 6 & 1 & & & & & & & & & \\
7 & 12 & 36 & & & & & & & & \\
8 & 96 & 168 & 114 & 24 & 6 & & & & & \\
9 & 196 & 760 & 384 & 108 & 60 & 168 & 108 & 96 & 12 & 4 \\
\hline
\end{tabular}


Table 5. $\mathcal{V}\left(j, H \square \sigma_{2}, t\right)$

\begin{tabular}{|c|c|c|c|c|c|c|c|c|c|c|}
\hline $\mathrm{j}$ & $B_{0}$ & $B_{1}$ & $B_{2}$ & $B_{3}$ & $B_{4}$ & $B_{5}$ & $B_{6}$ & $B_{7}$ & $B_{8}$ & $B_{9}$ \\
\hline $10 / 3$ & 1 & & & & & & & & & \\
$11 / 3$ & 4 & 12 & & & & & & & & \\
$12 / 3$ & 24 & 24 & 6 & & & & & & & \\
$13 / 3$ & 12 & 96 & 24 & 12 & & & & & & \\
$14 / 3$ & 78 & 192 & 120 & 24 & 6 & 12 & & & & 1 \\
$15 / 3$ & 144 & 312 & 192 & 24 & 28 & 84 & 48 & 36 & & \\
\hline
\end{tabular}

Table 6. $\mathcal{V}\left(j, H \square \sigma_{3}, t\right)$

\begin{tabular}{|c|c|c|c|c|c|c|c|c|c|c|}
\hline $\mathrm{j}$ & $B_{0}$ & $B_{1}$ & $B_{2}$ & $B_{3}$ & $B_{4}$ & $B_{5}$ & $B_{6}$ & $B_{7}$ & $B_{8}$ & $B_{9}$ \\
\hline $6 / 2$ & 5 & 4 & & & & & & & & \\
$7 / 2$ & 24 & 72 & 12 & & & & & & & \\
$8 / 2$ & 120 & 264 & 138 & 48 & 15 & 12 & 12 & & & \\
\hline
\end{tabular}

Table 7. $\mathcal{V}(j, H \square J, t)$

\begin{tabular}{|c|c|c|c|c|c|c|c|c|c|c|}
\hline $\mathrm{j}$ & $B_{0}$ & $B_{1}$ & $B_{2}$ & $B_{3}$ & $B_{4}$ & $B_{5}$ & $B_{6}$ & $B_{7}$ & $B_{8}$ & $B_{9}$ \\
\hline $10 / 6$ & 1 & & & & & & & & & \\
$11 / 6$ & & 8 & & & & & & & & \\
$12 / 6$ & 24 & 12 & 6 & & 1 & & & & & \\
$13 / 6$ & 24 & 96 & 12 & & & & 8 & & & \\
\hline
\end{tabular}

From these expansions we see that $\phi_{H}^{*} f$ vanishes to order at least 6 at every cusp so that there are pararmeters $\alpha_{0}, \ldots, \alpha_{8}$ and $\beta_{0}, \ldots, \beta_{7} \in \mathbb{C}$ such that

$$
\phi_{H}^{*} f=(D)^{6}\left(\alpha_{0} A^{8}+\alpha_{1} A^{7} B+\cdots+\alpha_{8} B^{8}+C\left(\beta_{0} A^{7}+\beta_{1} A^{6} B+\cdots+\beta_{7} B^{7}\right)\right) .
$$

Without introducing any new parameters we also have equalities for any $\sigma \in \Gamma_{1}$ :

$$
\begin{aligned}
\phi_{H}^{*} f \mid \sigma=(D \mid \sigma)^{6}\left(\alpha_{0}(A \mid \sigma)^{8}+\ldots\right. & +\alpha_{8}(B \mid \sigma)^{8} \\
& \left.+(C \mid \sigma)\left(\beta_{0}(A \mid \sigma)^{7}+\ldots+\beta_{7}(B \mid \sigma)^{7}\right)\right) .
\end{aligned}
$$

For $\sigma=I, \sigma_{2}, \sigma_{3}, J$ the left side of equation 3.2 is computed from equation 3.1, equation 1.2 and Tables 4 through 7 . The right side is computed from the expansions of the elliptic modular forms $A, B$ and $C$. At the cusp $[I]$ we equate the coefficents for 
$j=6, \ldots, 9$; at the cusp $\left[\sigma_{2}\right]$ for $j=6 / 3, \ldots, 15 / 3$; at the cusp $\left[\sigma_{3}\right]$ for $j=6 / 2, \ldots, 8 / 2$ and at the cusp $[J]$ for $j=6 / 6, \ldots, 13 / 6$. Elimination of the 17 parameters $\alpha_{i}, \beta_{i}$ from the $4+10+3+8=25$ linear equations results in 8 linearly independent equations:

$$
\begin{aligned}
& a\left(B_{2}\right)=-86 / 21 a\left(B_{0}\right)-188 / 21 a\left(B_{1}\right) \\
& a\left(B_{3}\right)=100 / 3 a\left(B_{0}\right)+58 / 3 a\left(B_{1}\right) \\
& a\left(B_{4}\right)=-300 / 7 a\left(B_{0}\right)+24 / 7 a\left(B_{1}\right) \\
& a\left(B_{5}\right)=-1892 / 21 a\left(B_{0}\right)+568 / 21 a\left(B_{1}\right) \\
& a\left(B_{6}\right)=288 / 7 a\left(B_{0}\right)-53 / 7 a\left(B_{1}\right) \\
& a\left(B_{7}\right)=2860 / 63 a\left(B_{0}\right)-8738 / 63 a\left(B_{1}\right) \\
& a\left(B_{8}\right)=656 / 7 a\left(B_{0}\right)+3872 / 7 a\left(B_{1}\right) \\
& a\left(B_{9}\right)=21016 / 21 a\left(B_{0}\right)+15532 / 21 a\left(B_{1}\right) .
\end{aligned}
$$

When we combine these 8 linear relations with the 2 linear relations in equation 1.5 obtained by considering $\phi_{D_{4}}^{*}$, we see that the rank is actually 9 , so that we have a total of 9 linearly independent relations in $a\left(B_{0}\right), \ldots, a\left(B_{9}\right)$ :

$$
\begin{aligned}
& a\left(B_{1}\right)=2 a\left(B_{0}\right) \\
& a\left(B_{2}\right)=-22 a\left(B_{0}\right) \\
& a\left(B_{3}\right)=72 a\left(B_{0}\right) \\
& a\left(B_{4}\right)=-36 a\left(B_{0}\right) \\
& a\left(B_{5}\right)=-36 a\left(B_{0}\right) \\
& a\left(B_{6}\right)=26 a\left(B_{0}\right) \\
& a\left(B_{7}\right)=-232 a\left(B_{0}\right) \\
& a\left(B_{8}\right)=1200 a\left(B_{0}\right) \\
& a\left(B_{9}\right)=2480 a\left(B_{0}\right) .
\end{aligned}
$$

These relations and Theorem 1.1 imply that $\operatorname{dim} S_{4}^{10} \leqslant 1$. Since we can come up with one nonzero cusp form $G_{10}$ in $S_{4}^{10}$ we have a theorem.

TheOREM 3.4. We have $\operatorname{dim} S_{4}^{10}=1$ and $S_{4}^{10}=\mathbb{C} G_{10}$.

\section{Final Comments}

The computations that have been performed for the form $H$ are largely independent of the weight $k$. Applied to the space $S_{4}^{8}$ we may extend the Fourier expansion of the Schottky form $J$ beyond that given in [1]. Table 8 gives the Fourier coefficients $a\left(B_{i}\right)$ for $J / 2^{16}$ and $G_{10} / 2^{18} 3^{4} 5$. 
Table 8. (Fourier Coefficients)

\begin{tabular}{|c|c|c|c|c|c|c|c|c|c|c|}
\hline$f$ & $B_{0}$ & $B_{1}$ & $B_{2}$ & $B_{3}$ & $B_{4}$ & $B_{5}$ & $B_{6}$ & $B_{7}$ & $B_{8}$ & $B_{9}$ \\
\hline$J / 2^{16}$ & 1 & -1 & 2 & 6 & -12 & -12 & 11 & 2 & -72 & 116 \\
$G_{10} / 2^{18} 3^{4} 5$ & 1 & 2 & -22 & 72 & -36 & -36 & 26 & -232 & 1200 & 2480 \\
\hline
\end{tabular}

Although the parameters $\alpha_{i}$ and $\beta_{i}$ were simply eliminated in Section 3, their values are also determined by this process. It may be of interest to present the images of $\phi_{s}^{*} f$ for $s=D_{4}, H$ and $f=J, G_{10}$.

$$
\begin{aligned}
\phi_{D_{4}}^{*} J & =2^{16}\left(C_{8,2}^{+}\right)^{4} \\
\phi_{D_{4}}^{*} G_{10} & =2^{18} 3^{4} 5\left(C_{8,2}^{+}\right)^{4}\left(\left(E_{2,2}^{-}\right)^{4}+48 C_{8,2}^{+}\right) \\
\phi_{H}^{*} J & =2^{12} D^{6}(A+C)^{4} \\
\phi_{H}^{*} G_{10} & =2^{14} 3^{3} 5 D^{6}(A+C)^{4}\left(25 A^{4}-8 A^{3} B-7 A^{3} C-8 A^{2} B C-A B C^{2}+4 A C^{3}-B C^{3}-C^{4}\right)
\end{aligned}
$$

It is interesting to note that the image of $G_{10}$ comes out to a multiple of the image of $J$ under both $\phi_{D_{4}}^{*}$ and $\phi_{H}^{*}$. As a final comment we note that linear relations among Fourier coefficients can be viewed as linear relations among Poincare series.

\section{REFERENCES}

[1] B. Brinkman and L. Gerritzen, 'The lowest term of the Schottky modular form', Math. Annalen 292 (1992), 329-335.

[2] U. Christian, Selberg's Zeta-, L-, and Eisenstein series, Lecture Notes in Math. 1030 (Springer-Verlag, Berlin, Heidelberg, New York, 1983).

[3] W. Duke and Ö. Imamoḡlu, 'Siegel modular forms of small weight', Math. Ann. 308 (1997), 525-534.

[4] M. Eichler, "Über die Anzahl der linear unabhängigen Siegelschen Modulformen von gegebenem Gewicht', Math. Ann. 213 (1975), 281-291.

[5] M. Eichler, 'Erratum: Über die Anzahl der linear unabhängigen Siegelschen Modulformen von gegebenem Gewicht', Math. Ann. 215 (1975), 195.

[6] V.A. Erokhin, 'Theta series of even unimodular 24-dimensional lattices', Zap. Naučn. Sem. Leningrad. Otdel. Mat. Inst. Steklov. (LOMI) 86 (1979), 82-93.

[7] V.A. Erokhin, 'Theta series of even unimodular lattices', Zap. Nauchn. Sem. Leningrad. Otdel. Mat. Inst. Steklov. (LOMI) 112 (1981), 59-70.

[8] E. Freitag, Siegelsche Modulfunktionen, Grundlehren der mathematische Wissenschaften 254 (Springer Verlag, Berlin, 1983).

[9] J.I. Igusa, 'Schottky's invariant and quadratic forms', in Christoffel Symposium (Birkhäuser Verlag, Basel, Boston, MA, 1981), pp. 352-362.

[10] G. Nipp, Quaternary quadratic forms, computer generated tables (Springer-Verlag, New York, 1991). 
[11] C. Poor and D. Yuen, 'Dimensions of spaces of Siegel modular forms of low weight in degree four', Bull. Austral. Math. Soc. 54 (1996), 309-315.

[12] C. Poor and D. Yuen, 'Dimensions of spaces of Siegel cusp forms and theta series with pluri-harmonics', Far East J. Math. Sci. (FJMS) 1 (1999), 849-863.

[13] C. Poor and D. Yuen, 'Linear dependence among Siegel Modular Forms', Math. Ann. 318 (2000), 205-234.

[14] R. Salvati Manni, 'Modular forms of the fourth degree (Remark on a paper of Harris and Morrison)', in Classification of irregular varieties, (Ballico, Catanese, Ciliberto, Editors), Lecture Notes in Math. 1515 (Springer, Berlin, 1992), pp. 106-111.

Department of Mathematics

Fordham University

Bronx, NY 10458

e-mail: poor@fordham.edu
Math/CS Department

Lake Forest College

555 N. Sheridan Rd.

Lake Forest, IL 60045

e-mail: yuen@lfc.edu 